\title{
PIK3CA NP_006209.2:p.H1047Y
}

National Cancer Institute

\section{Source}

National Cancer Institute. PIK3CA NP 006209.2:p.H1047Y. NCI Thesaurus. Code C98487.

A change in the amino acid residue at position 1047 in the phosphatidylinositol-4,5bisphosphate 3-kinase catalytic subunit alpha isoform protein where histidine has been replaced by tyrosine. 